\title{
Theoretical Study on Molecular Structure and Electronic Properties of New 1,3-Diaza-adamantan-6-ones Derivatives
}

\author{
Haithem Abdulhasan, Ahmed Al-Yasari ${ }^{*}$, and Rahman Alasadi \\ University of Kerbala, P.O. Box 1125, Kerbala, Iraq
}

${ }^{*}$ Corresponding author:

tel: +96432-7729137527

email:a.alyasari@uokerbala.edu.iq

Received: March 22, 2019

Accepted: May 14, 2019

DOI: $10.22146 /$ ijc.44403

\begin{abstract}
In this study, the structural geometry and vibrational frequencies (IR) of 1,3Diaza-adamantane-6-ones derivatives including Adamantane (A), 1,3-Diazaadamantan (D), 1,3-Diaza-adamantan-6-one (DO), 5-Benzyl-1,3-diaza-adamantan-6one (BD), 5-(4-Hydroxybenzyl)-1,3-diaza-adamantan-6-one (HBD), 5-(4-Methoxy benzyl)-1,3-diaza-adamantan-6-one (MBD), and 5-(4-Hydroxy-3-methoxybenzyl)-1,3diaza-adamantan-6-one (HMBD) were theoretically studied. In addition, molecular orbital energies, including the highest occupied molecular orbitals (HOMOs), and lowest unoccupied molecular orbitals (LUMOs), and electronic properties of the titled molecules were theoretically studied using the computational method. Optimized molecular structures were obtained by DFT method with the hybrid B3LYP functional at a relatively small basis set of 6-31G. The calculated vibrational wavenumbers were obtained using the same level of the theory mentioned above. The contributions to the molecular orbitals of adamantane and substituted-phenyl groups in the title compounds were determined. Moving from A to HMBD, a decrease in the value of LUMO and total energy are noticed, while an increase in the value of HOMO is noted. These findings are supported by the decrease in the $E_{\text {Номо-цим }}$ gap values. Furthermore, a decrease in the value of ionization potential (IP) is obtained, while an increase in the electron affinity (EA) is observed.
\end{abstract}

Keywords: Adamantane; 1,3-Diaza-adamantan; DFT/B3LYP

\section{- INTRODUCTION}

Adamantane and its derivatives have fascinating structures due to their various physiological, pharmaceutical, and medical activities [1-6]. It consists of a polycyclic cage molecule with high symmetry representing diamondoids-hydrogen-terminated hydrocarbons with a diamond-like structure [7-8]. Among these derivatives, 1,3-Diaza-adamantane derivatives have attracted great attention due to their interesting properties [9] such as antibacterial, and psychotropic activity [10-11]. SharabiRonen et al. have reported the anti-neoplastic activity of 1,3-Diaza-2-functionalized-adamantan-6-one compounds against melanoma cells [12]. They showed that the ability of the reported compounds to introduce apoptosis in melanoma cells was significant.

1,3-Diazaadamantane-6-one derivatives have attracted considerable attention for diverse applications. Recently, we reported the synthesis of a new series of 1,3-
Diaza-adamantane-6-ones derivatives [13]. However, their structural geometry and vibrational properties have not been studied yet. To the best of our knowledge, the quantum chemical calculations on these derivatives have not been done so far. In this study, the molecular structure and the full vibrational spectra have been reported. Moreover, the effect of substitution on electronic properties such as the ionization potential, the electron affinity, electronegativity, and the energy gap between HOMO-LUMO are generally studied.

\section{- COMPUTATIONAL METHODS}

All computational procedures were carried out using GAMESS and Avogadro 1.1.1 [14-15]. The optimization structure of Adamantane (A), including 1,3-Diaza-adamantan (D), 1,3-Diaza-adamantan-6-one (DO), 5-Benzyl-1,3-diaza-adamantan-6-one (BD), 5-(4Hydroxybenzyl)-1,3-diaza-adamantan-6-one (HBD), 5-

Haithem Abdulhasan et al. 
(4-Methoxybenzyl)-1,3-diaza-adamantan-6-one (MBD) and 5-(4-Hydroxy-3-methoxybenzyl)-1,3-diaza-adaman tan-6-one (HMBD) molecules have been performed using DFT method with the hybrid B3LYP functional at 6-31G level in the gas phase. The applied method is a combination of the Hartree-Fock method and the density functional theory using Becke's three parameter (B3) gradient corrected functionals [15] and Lee-Yang exchange-correlation functional (LYP) [16]. Molecular geometries of all systems were fully optimized at the B3LYP functional with a relatively small basis set of 6-31G level of theory. The stationary points determined with geometry optimization with minimal energy was confirmed by calculating the normal vibration frequencies with the use of second derivatives at the same level of theory. The energy gap (Е Еомо-Luмо gap), Ionization potential (IP), electron affinity (EA), and the electronegativity (X) have been calculated using equations $1,2,3,4$, respectively.

$\mathrm{E}_{\text {HOMO-LUMO }}$ gap $=\mathrm{E}_{\text {LUMO }}-\mathrm{E}_{\mathrm{HOMO}}$

$\mathrm{IP}=-\mathrm{E}_{\mathrm{HOMO}}$

$\mathrm{EA}=-\mathrm{E}_{\mathrm{LUMO}}$

$\mathrm{X}=-0.5\left(\mathrm{E}_{\mathrm{HOMO}}+\mathrm{E}_{\mathrm{LUMO}}\right)$

Both the vibrational modes and frequencies were assigned, and the animations of normal modes were visualized by using Avogadro 1.1.1 [17].
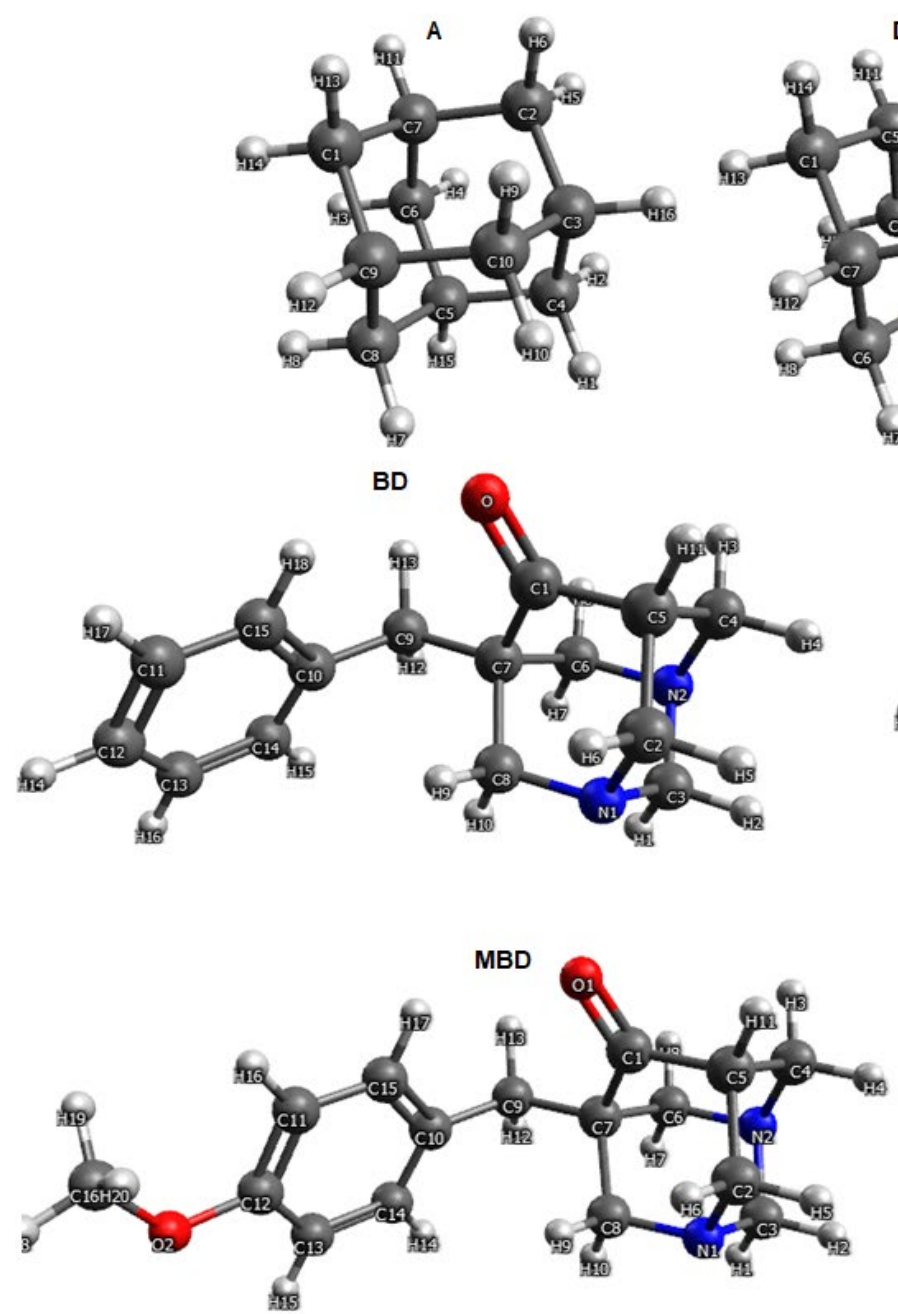
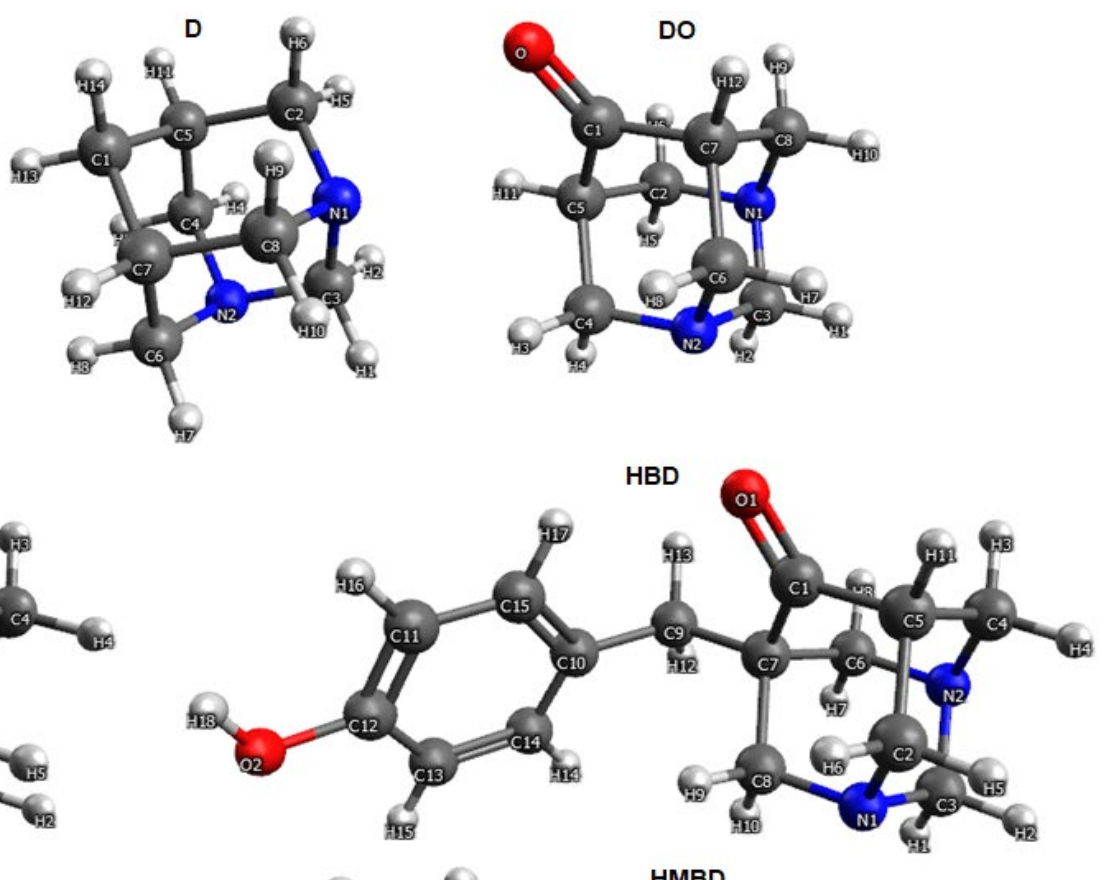

HMBD

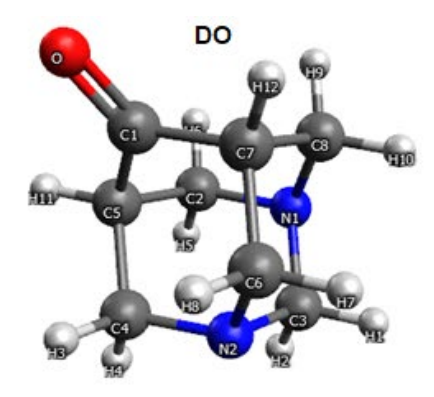

Fig 1. The optimized structure of molecules A, D, DO, BD, HBD, MBD, and HMBD 


\section{- RESULTS AND DISCUSSION}

\section{Molecular Geometric Structure}

The chemical reactivity and hence, the chemical properties are affected by the change in molecular structure. Therefore, the effect of substitution of 1,3diaza-adamantan derivatives with their parent Adamantane (A), including 1,3-Diaza-adamantan (D), 1,3-Diaza-adamantan-6-one (DO), 5-Benzyl-1,3-diazaadamantan-6-one (BD), 5-(4-Hydroxybenzyl)-1,3-diazaadamantan-6-one (HBD), 5-(4-Methoxybenzyl)-1,3diaza-adamantan-6-one (MBD) and 5-(4-Hydroxy-3methoxybenzyl)-1,3-diaza-adamantan-6-one (HMBD) have been studied.

Fig. 1 shows the geometric structures of the asstudied molecules as obtained by calculations. Selections of the most important structural parameters of all molecules are listed in Table 1, and the full details of geometric parameters may be obtained from the author upon request. By considering the data for compound $\mathbf{A}$ and $\mathbf{D}$, upon the substitution of two carbon by two nitrogen, the latter showed an increase in the angle value of N1-C3$\mathrm{N} 2$ due to the effect of lone pair in the nitrogen of the heteroatom. Generally, in all compounds, N-C-N angles are slightly wider than $\mathrm{C}-\mathrm{N}-\mathrm{C}$ angles. In the case of compound HMBD, the bond length of $\mathrm{C} 12-\mathrm{O} 2$ is shorter than that in compound MBD, but the length of $\mathrm{O} 3-\mathrm{C} 16$ is longer. The C7-C9-C10 angle is slightly wider compared with compound MBD, and noticeably wider than the other studied compounds.

The same trend was noticed for the value of C5C1-C7. The C4-N2-C6 showed a slight increase in its value when moving from compound $\mathrm{D}$ to HMBD. The

Table 1. Selected geometric parameters (bond distances, $\AA$, bond and torsion angles, ${ }^{\circ}$ ) of A, D, DO, BD, HBD, MBD, and HMBD

\begin{tabular}{lccccccc}
\hline & & & & & & \\
& & & & & & &
\end{tabular}

Haithem Abdulhasan et al. 
same trend was observed in the $\mathrm{C} 2-\mathrm{N} 1-\mathrm{C} 8$. The $\mathrm{C} 1-\mathrm{O} 1 \mathrm{in}$ compound DO is noticeably shorter than in all compounds due to the effect of the phenyl group in these compounds. The same was noticed for the $\mathrm{N}-\mathrm{C}$ bond length, where it is remarkably shorter in DO compared to the others. The negative hyper-conjugative effect of lone pair in the nitrogen atom toward the carbonyl contributed to the decrease in the $\mathrm{N}-\mathrm{C}$ bond lengths. These results are in line with those observed in earlier studies by JiménezCruz et al. [18].

\section{Vibrational Frequencies}

The IR spectra of molecules were calculated in the range of $0-4000 \mathrm{~cm}^{-1}$. Comparison of the calculated and experimental results show a relatively good agreement despite that the experimental values were recorded in the solid state while the calculated values were studied in the gas phase [13]. However, overestimation of some frequencies was noticed, such as the $v \mathrm{O}-\mathrm{H}$ stretching of HBD. Future works are needed to address these overestimations and to investigate further effects such as solvation. Table 2 shows the IR data of the experimental and calculated values of studied molecules. In the case of compound BD that was chosen as a model of these series, experimentally [13] absorption bands were shown at $2952 \mathrm{~cm}^{-1}$ ( $v$ C-H, benzene), $1464 \mathrm{~cm}^{-1}$ ( $v \mathrm{C}=\mathrm{C}$, benzene), $880 \mathrm{~cm}^{-1}$ ( $v \mathrm{C}-\mathrm{H}$, benzene), $1707 \mathrm{~cm}^{-1}(\nu \mathrm{C}=\mathrm{O}$, ketone), $1605 \mathrm{~cm}^{-1}(v \mathrm{Ph})$, which almost coincide with those of maxima obtained theoretically, as shown in Fig. 2.

Generally, in the region of $1680-1730 \mathrm{~cm}^{-1}$, the carbonyl $\mathrm{C}=\mathrm{O}$ stretching vibrations are observed. In the case of compound BD, this band was obtained as a strong band at $1689 \mathrm{~cm}^{-1}$. The band of stretching phenyl ( $\left.\nu \mathrm{Ph}\right)$ group was noticed in the region of $1550-1650 \mathrm{~cm}^{-1}$. Calculated spectra of the studied compounds can be provided upon request.

\section{Molecular Orbital Energies (HOMO and LUMO Analyses)}

The frontier molecule orbitals (FMOs) which refer to the HOMOs and LUMOs, have an important role in

Table 2. FT-IR data of the experimental [13] (and calculated) values of BD, HBD, MBD, and HMBD

\begin{tabular}{lcccc}
\hline Compound & $v \mathrm{C}=\mathrm{O}$, ketone $/ \mathrm{cm}^{-1}$ & $v \mathrm{Ph} / \mathrm{cm}^{-1}$ & $v \mathrm{O}-\mathrm{H} / \mathrm{cm}^{-1}$ & $v \mathrm{OCH} 3 / \mathrm{cm}^{-1}$ \\
\hline BD & 1707 & 1605 & - & - \\
& $(1689)$ & $(1637)$ & & - \\
HBD & 1704 & 1600 & 3200 & \\
& $(1686)$ & $(1646)$ & $(3600)$ & 1251,1127 \\
MBD & 1713 & 1609 & - & $(1250,1172)$ \\
& $(1682)$ & $(1632)$ & & 1270,1145 \\
HMBD & 1706 & 1569 & 3396 & $(1270,1168)$ \\
& $(1680)$ & $(1561)$ & $(3600)$ & \\
\hline
\end{tabular}

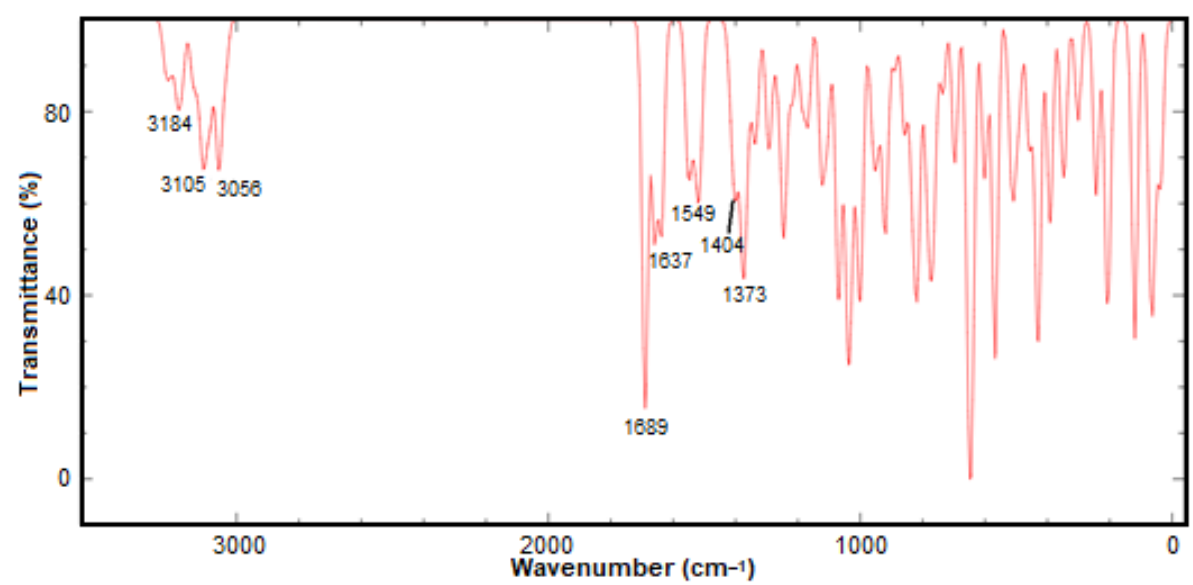

Fig 2. Calculated IR spectra of BD in the frequency range of $0-3300 \mathrm{~cm}^{-1}$ 
chemical reactions, molecular electronic and physiochemical properties such as ionization potential, electron affinity, chemical reactivity, kinetic stability, electronegativity, and electrophilicity [19-20]. The calculated HOMOs and LUMOs of the titled molecules are shown in Fig. 3. Table 3 shows the electronic characteristics calculated for the given molecules: the frontier orbital energies $\left(\mathrm{E}_{\text {homo, }}, \mathrm{E}_{\text {lumo }}\right)$, the zero-point energy (ZPE), and the entropy ( $\mathrm{S}$ ) and final total energy
$\left(\mathrm{E}_{\text {final }}\right)$. Generally, the results showed a decrease in final energy of optimized structures as follows: A $>$ D $>$ DO $>$ BD $>$ HBD $>$ MBD $>$ HMBD. The results also showed the same trend in the energy of $\mathrm{E}_{\mathrm{HOMO}}$ and $\mathrm{E}_{\mathrm{LUMO}}$; an increase in the energy of $\mathrm{E}_{\text {номо }}$ and a decrease in the energy of $\mathrm{E}_{\mathrm{LUmo}}$. This is attributed to the effect of the replacement of carbon atoms with good donor atoms such as nitrogen and oxygen through comparison between molecules $\mathbf{A}$ and $\mathbf{D}$.
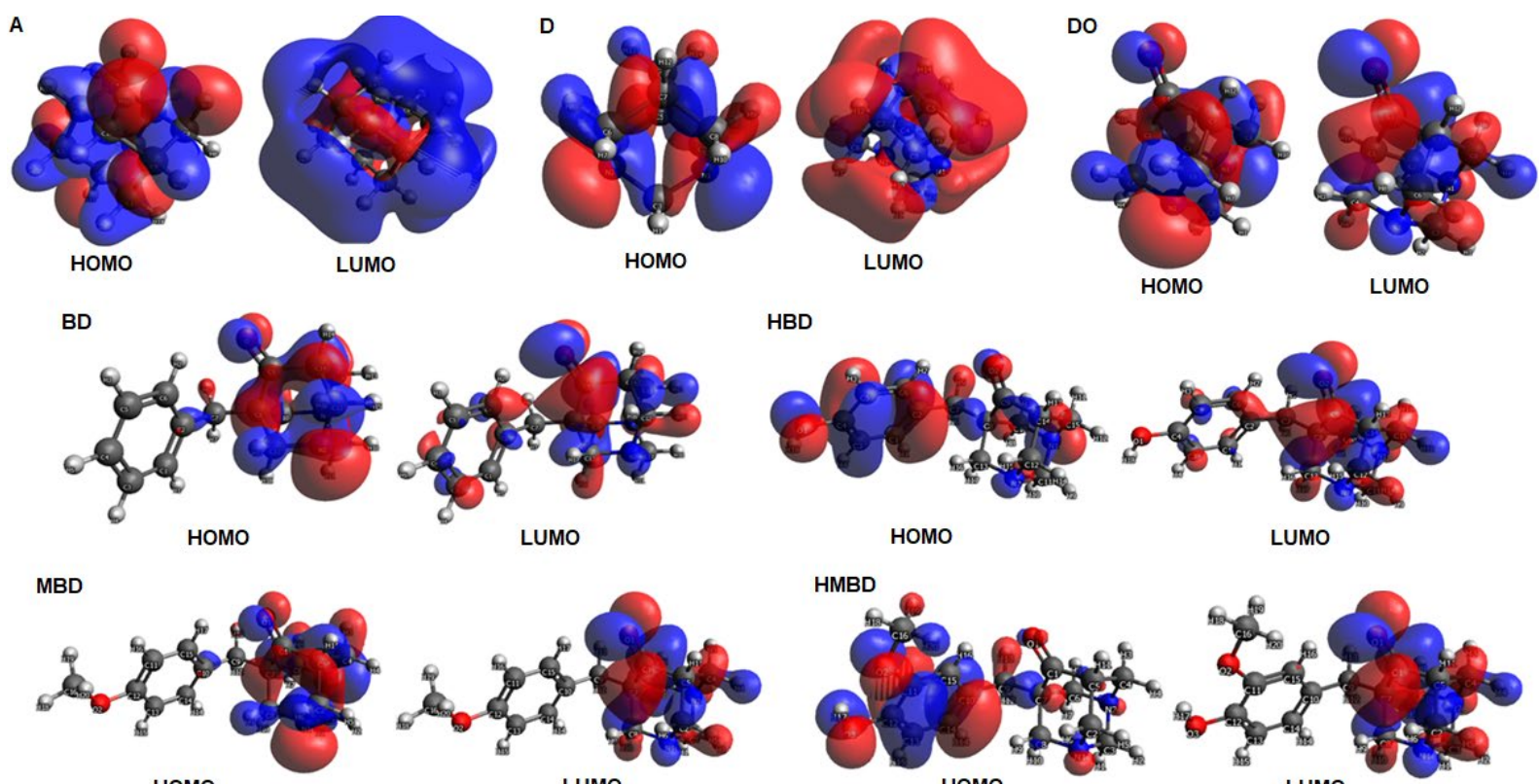

номо

LUMO

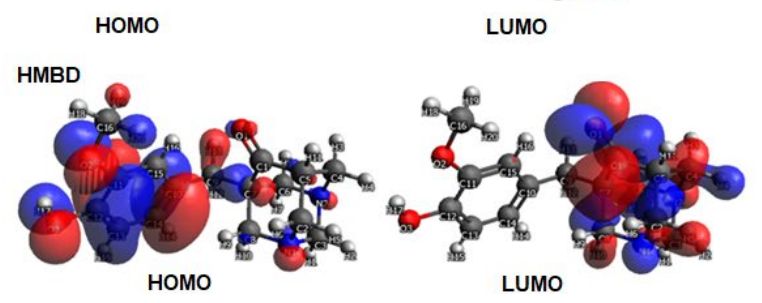

Fig 3. Frontier molecular orbitals of A, D, DO, BD, HBD, MBD, and HMBD molecules

Table 3. Calculated basic electronic energy characteristics of the studied compounds at room temperature $(298.15 \mathrm{~K})$

\begin{tabular}{|c|c|c|c|c|c|}
\hline Compound & $\mathrm{E}_{\text {final, }}$ a.u. $\left(\mathrm{E}_{\text {final }} \mathrm{kcal} / \mathrm{mol}\right)$ & $\mathrm{E}_{\text {Hомо }}, \mathrm{eV}$ & $\mathrm{E}_{\mathrm{LUMO}}, \mathrm{eV}$ & $\mathrm{S}, \mathrm{cal} /(\mathrm{mol} \mathrm{K})$ & ZPE, $\mathrm{kcal} / \mathrm{mol}$ \\
\hline \multirow[t]{2}{*}{ A } & -390.348 & -7.525 & 2.414 & 81.192 & 154.291 \\
\hline & $(-244947.53)$ & & & & \\
\hline \multirow[t]{2}{*}{ D } & -422.359 & -5.217 & 1.215 & 80.259 & 139.339 \\
\hline & $(-265034.71)$ & & & & \\
\hline \multirow[t]{2}{*}{ DO } & -496.320 & -5.797 & -0.358 & 85.088 & 126.765 \\
\hline & $(-311446.10)$ & & & & \\
\hline \multirow[t]{2}{*}{ BD } & -766.448 & -5.784 & -0.526 & 114.764 & 196.305 \\
\hline & $(-480954.36)$ & & & & \\
\hline \multirow[t]{2}{*}{ HBD } & -841.608 & -5.745 & -0.478 & 119.739 & 198.515 \\
\hline & $(-528117.68)$ & & & & \\
\hline \multirow[t]{2}{*}{ MBD } & -880.873 & -5.721 & -0.451 & 126.422 & 216.776 \\
\hline & $(-552756.73)$ & & & & \\
\hline \multirow[t]{2}{*}{ HMBD } & -956.036 & -5.382 & -0.492 & 132.372 & 219.081 \\
\hline & $(-599922.67)$ & & & & \\
\hline
\end{tabular}


Table 4. Ionization potential (IP), electron affinity (EA) and electronegativity (X) values of the studied compounds

\begin{tabular}{lcrrl}
\hline Compound & E $_{\text {Hомо-Lumo gap/eV }}$ & $\mathrm{IP} / \mathrm{eV}$ & $\mathrm{EA} / \mathrm{eV}$ & $\mathrm{X} / \mathrm{eV}$ \\
\hline A & 9.939 & 7.525 & -2.414 & 2.5555 \\
D & 6.432 & 5.217 & -1.215 & 2.001 \\
DO & 5.439 & 5.797 & 0.358 & 3.0775 \\
BD & 5.258 & 5.784 & 0.526 & 3.155 \\
HBD & 5.267 & 5.745 & 0.478 & 3.1115 \\
MBD & 5.27 & 5.721 & 0.451 & 3.086 \\
HMBD & 4.89 & 5.382 & 0.492 & 2.937 \\
\hline
\end{tabular}

When comparing molecule $\mathbf{D}$ with $\mathbf{D O}$, where $\mathrm{C}=\mathrm{O}$

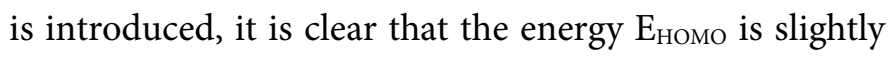
decreased. While in the case of molecules BD, HBD, MBD, HMBD compared with molecule A, changes in the energy of $\mathrm{E}_{\mathrm{HOMO}}$ and $\mathrm{E}_{\mathrm{LUMO}}$ are the result from the introduction of the $\pi$-system (phenyl group) and donor groups. It is well known that the high value of the $\mathrm{E}_{\mathrm{Hомо}}$ indicates a strong donor molecule, while the low value of the $\mathrm{E}_{\mathrm{LUMO}}$ is likely referring to a good acceptor molecule to form stable bonds.

\section{Electronic Properties: HOMO-LUMO Gap Energy (Eномо-цuмо Gap), Electron Affinity (EA), Ionization Potential (IP), and Electronegativity (X)}

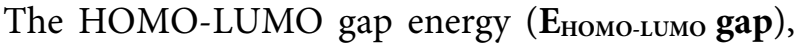
Ionization potential (IP), electron affinity (EA) and electronegativity $(\mathbf{X})$ were calculated by using the B3LYP with 6-31G level of theory for the titled molecules, as described in Computational Methods. Table 4 presents the calculated HOMO-LUMO gap energy ( $\mathbf{E}_{\text {номо-Lumo }}$ gap), Ionization potential (IP), electron affinity (EA), and electronegativity $(\mathbf{X})$ values of the titled molecules calculated using equations 1-4. Generally, when comparing the titled molecules, the $\mathrm{E}_{\text {номо-цuм }}$ gap is changed as follows: $\mathbf{A}>\mathbf{D}>\mathbf{D O}>\mathbf{B D}<\mathbf{H B D}<$ MBD $>$ HMBD. It is known that IPs and EAs are closely related to the energies of HOMO and LUMO, respectively. It can be seen from Table 4 that the highest IP value is for $\mathbf{A}$ indicating the more stable molecule with inertness in chemical reactivity. Meanwhile, the lowest IP value is for both $\mathrm{D}$ and HMBD due to the effect of the donor groups in these molecules, indicating more reactive molecules.
The calculated EA values of compound $\mathbf{A}$ and $\mathbf{D}$ are negative while for the rest of molecules are positive. A close look at these results, molecule $\mathbf{A}$ is more negative than that of molecule $\mathrm{D}$. In the case of BD, HBD, MBD, and HMBD, the EA values are positive due to the presence of donor groups. From Table 4, it can also be noticed that the electronegativity $(\mathbf{X})$ of the titled molecules are high (positive). These findings are important in many applications, such as corrosion inhibition. Further work is needed to investigate the properties of these materials as a corrosion inhibitor.

\section{- CONCLUSION}

To sum up, the structure and physiochemical properties of Adamantane (A), 1,3-Diaza-adamantan (D), 1,3-Diaza-adamantan-6-one (DO), 5-Benzyl-1,3-diazaadamantan-6-one (BD), 5-(4-Hydroxybenzyl)-1,3-diaza -adamantan-6-one (HBD), 5-(4-Methoxy benzyl)-1,3diaza-adamantan-6-one (MBD), and 5-(4-Hydroxy-3methoxybenzyl)-1,3-diaza-adamantan-6-one (HMBD) have been revealed at a relatively small basis set by using the DFT calculations with the hybrid B3LYP functional and 6-31G level of theory. The IR spectra of the titled molecules have been calculated in the gas phase. Moving from $\mathbf{A}$ to HMBD, a decrease in the values of LUMO and total energy are noticed, while the values of HOMO increase, indicating the more reactive molecule in the reactions with electrophiles with potential application as a corrosion inhibitor. These findings are supported by

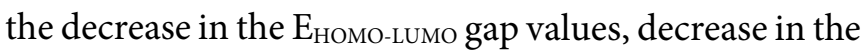
values of IP, and increase in the values of EA.

\section{- REFERENCES}

[1] Štimac, A., Šekutor, M., Mlinarić-Majerski, K., Frkanec, L., and Frkanec, R., 2017, Adamantane in drug delivery systems and surface recognition, Molecules, 22 (2), 297.

[2] Savel'eva, S.A., Leonova, M.V., Baimuratov, M.R., and Klimochkin, Y.N., 2018, Synthesis and transformations of aryl-substituted alkenes of the adamantane series, Russ. J. Org. Chem., 54 (7), 9961002 .

[3] Ivleva, E.A., Tkachenko, I.M., and Klimochkin, Y.N., 
2017, Synthesis of adamantane functional derivatives basing on $\mathrm{N}-[($ adamantan-1-yl)alkyl]acetamides, Russ. J. Org. Chem., 52 (11), 1558-1564.

[4] Suslov, E., Zarubaev, V.V., Slita, A.V., Ponomarev, K., Korchagina, D., Ayine-Tora, D.M., Reynisson, J., Volcho, K., and Salakhutdinov, N., 2017, Antiinfluenza activity of diazaadamantanes combined with monoterpene moieties, Bioorg. Med. Chem. Lett., 27 (19), 4531-4535.

[5] Hickmott, P.W., Wood, S., and Murray-Rust, P., 1985, Introduction of pharmacophoric groups into polycyclic systems. Part 3. Amine derivatives of adamantane and diaza-adamantane, J. Chem. Soc., Perkin Trans. 1, 0, 2033-2038.

[6] Vrynchanu, N.A., Sergienko, O.V., and Maksimov Yu, N., 2009, Research of some sides of antifungal activity mechanism act of new adamantane derivative, Морбологія, 3 (2), 24-27.

[7] Schwertfeger, H., Fokin, A.A., and Schreiner, P.R., 2008, Diamonds are a chemist's best friend: Diamondoid chemistry beyond adamantane, Angew. Chem. Int. Ed., 47 (6), 1022-1036.

[8] Gunawan, M.A., Hierso, J.C., Poinsot, D., Fokin, A.A., Fokina, N.A., Tkachenko, B.A., and Schreiner, P.R., 2014, Diamondoids: Functionalization and subsequent applications of perfectly defined molecular cage hydrocarbons, New J. Chem., 38 (1), 28-41.

[9] Karthik, G., Sundaravadivelu, M., Rajkumar, P., and Manikandan, M., 2014, Diaza-adamantane derivatives as corrosion inhibitor for copper in nitric acid medium, Res. Chem. Intermed., 41 (10), 7593-7615.

[10] Arutyunyan, G.L., Paronikyan, R.V., Saakyan, G.S., Arutyunyan, A.D., and Gevorkyan, K.A., 2008, Synthesis and reactions of polyhedral compounds. 29. Synthesis and antibacterial activity of 1,3diazaadamantane derivatives, Pharm. Chem. J., 42 (1), 18-22.

[11] Arutyunyan, G.L., Dzhagatspanyan, I.A., Nazaryan, I.M., Akopyan, A.G., and Arutyunyan, A.D., 2007, Synthesis and conversions of polyhedral compounds: 28. Synthesis and psychotropic activity of some 1,3- diazaadamantane derivatives, Pharm. Chem. J., 41 (11), 591-593.

[12] Sharabi-Ronen, Y., Levinger, S., Lellouche, M.B., and Albeck, A., 2014, Anti-neoplastic activity of 1,3-diaza-2-functionalized-adamantan-6-one com pounds against melanoma cells, Med. Chem., 10 (1), 27-37.

[13] Kuznetsov, A.I., Alasadi, R.T., Senan, I.M., and Serova, T.M., 2014, Synthesis of fragrant 1,3diazaadamantan-6-ones, Russ. Chem. Bull., 63 (9), 2195-2197.

[14] Gordon, M.S., and Schmidt, M.W., 2005, "Advances in electronic structure theory: GAMESS a decade later" in Theory and Applications of Computational Chemistry: The first forty years, Eds. Dykstra, C., Frenking, G., Kim, K., and Scuseria, G., $1^{\text {st }}$ Ed., Elsevier Science, Amsterdam, 1167-1189.

[15] Becke, A.D., 1993, A new mixing of Hartree-Fock and local density-functional theories, J. Chem. Phys., 98 (2), 1372.

[16] Fernández, M.J., Gálvez, E., Lorente, A., Camuñas, J.A., Sanz, J., and Fonseca, I., 1990, Synthesis, structural and conformational study of 6-hydroxy (or acyloxy) derivatives of the 1,3-dimethyl-1,3diazoniatricyclo[3.3.1.1 $1^{3-7}$ decane system, $J$. Heterocycl. Chem., 27 (5), 1355-1359.

[17] Hanwell, M.D., Curtis, D.E., Lonie, D.C., Vandermeersch, T., Zurek, E., and Hutchison, G.R., 2012, Avogadro: An advanced semantic chemical editor, visualization, and analysis platform, 2012, J. Cheminform., 4 (1), 17.

[18] Jiménez-Cruz, F., Ríos-Olivares, H., and Gutiérrez, J.L.G., 2005, "Molecular structure in 1azaadamantanes and 1,3-diazaadamantanes" in Structural Analysis of Cyclic Systems, Eds. Iriepa, I., Research Signpost, Trivandum, India, 101-125.

[19] Parr, R.G., Donnelly, R.A., Levy, M., and Palke, W.E., 1978, Electronegativity: The density functional viewpoint, J. Chem. Phys., 68 (8), 3801.

[20] Figueredo, S., Páez, M., and Torresbc, F., 2019, The electrophilic descriptor, Comput. Theor. Chem., 1157, 34-39. 